about an hour. In this case, at all events, the mode of death was evidently predisposed to by the poisoned state of the blood.

III. We come at last to that part of the subject to which I wish more particularly to direct your attention - the death from Cardiac Syncope as it is called. This is to me a very puzzling mode of death, as difficult to account for as it is to guard against. I presume that by the term "cardiac syncope" is meant atony and failure of the heart's action. This is an easy explanation, but not altogether a satisfactory one. Amongst the numerous experiments which have been made with chloroform, I do not know of any which prove that it has any direct syncopal action on the heart, or even any indirect toxic action on that organ through its nerves. Still there is no doubt of the fact, that people do die without much disturbance of respiration, without becom. ing distinctly livid in the face ; the pulse fails; that is the first thing noticed, and they are dead.

Now my own idea is, that these are really cases of asphyxia ; that the heart is secondarily, not primarily, affected; and my explanation would be as follows. After death we find in these cases a weak, fatty heart ; the valves, indeed, healthy, but the walls thin, and the muscular tissue pale and degenerated. Now chloroform has always, especially at first, a slight asphyxial tendency; the patient calls out that he is choking, tries to pull away the inhaler, and breathes deeply, then struggles and holds his breath for a few seconds. In a healthy man this soon passes off, the inconvenience is merely temporary; but with a fatty, enfeebled heart, it is different-the patient holds his breath for a few seconds, the right side of the heart is soon filled, there is weak propulsive power, the organ cannot recover itself, and the result is fatal.

Some years ago, I made numerous experiments on death by asphyxia in animals ; and $I$ found that if once the ventricular action were stopped, if a contraction were missed, it was most difficult to start again-generally all was over. And that is what happens in these cases; the ven tricle cannot be emptied quickly enough, the rhythm is lost, and almost instantaneous death ensues. The patient, then, does not die from the direct action of the chloroform on the heart, but from the effect of a slight asphyxial condition, which is inseparable from the administration of this agent on a disorganised heart. After death, you may not find the right side of the heart greatly engorged ; first, because great engorgement is not necessary to cause a fatal result ; and, secondly, the blood, even many hours after death from chloroform, is found unusually fluid, so that the dependent parts of the body are congested and the heart is left comparatively empty. Then, also, artificial respiration is always set up in these cases, and this tends to diminish the cardiac plethora.

Finally, a few cautions. Nevergive chloroform without first thoroughly loosening the dress; if possible, not within four hours of a meal; the head should be moderately raised ; the pulse, respiration, and colour of the face, must be carefully watched. As regards the occurrence of the rigid spasm already adverted to, I do not think that sufficient attention has been directed to this condition. A patient with an enfeebled heart is then in a most dangerous, even critical, state ; the chest-walls are fixed, the lungs are filled with chloroform-vapour, which becomes diffused but cannot escape, the pulmonary circulation is obstructed, and the pressure on the right ventricle rapidly increased. Let the patient partially recover, then give it again slowly ; watch for any blueness around the mouth, etc. If the pulse fail, draw forward the tongue with forceps, and set up artificial respiration at once ; but prevention is the main point. When there is well marked asphyxia, artificial respiration is most useful, and generally successful ; but in these cases of so-called "cardiac syncope", it is generally useless ; the heart has been barely able to meet the ordinary requirements of the system ; it cannot recover lost ground ; it gives in, as it were, at once.

\section{STATISTICS OF OVARIOTOMY.}

IN the JOURNAL for May 18 th, are some valuable remarks on the statistics of ovariotomy. It is stated that up to November 1866, there were in King's College Hospital seven cases, with recovery and six deaths; being a mortality of 85.71 per cent. I do not know how many cases of ovariotomy have been performed up to the date given; but I do know that the following cases occurring in Sir William Fergusson's practice at that institution proved successful. February 1863, Emma R.; aged 26; February 1865, Esther R., aged 26; March 1865, Margaret C., aged I9. In the third case there were extensive adhesions. The cyst was not removed, but the opening into it stitched to the margins of the external wound. Suppuration took place, and the woman recovered after a severe illness. When she was seen a year after the operation, there was no return of the disease.

H. ROYES BELL, Assistant-Surgeon to King's College Hospital.

\section{THE ANATOMY OF BRIGHT'S DISEASE: \\ THE "ARTERIO-CAPILLARY FIBROSIS" OF SIR WM. GULL AND DR. SUTTON.}

By GEORGE JOHNSON, M.D., F.R.S., Physician to King's College Hospital ; Professor of Medicine in King's College ; etc.

IN consequence of Sir William Gull and Dr. Sutton having arranged not to exhibit their specimens at the recent meeting of the Royal Medical and Chirurgical Society, those who were present when their paper was read laboured under the great disadvantage of having to form a judgment upon an anatomical question without that appeal to the sense of vision by which alone the question could be decided. If I could have seen the specimens before I was called upon to discuss the statements put forward by the authors of the paper, my remarks would have been much more brief and decided than they were. After hearing the paper and looking carefully at the drawings, it was evident that there had been either a great discovery or a great mistake. Having worked steadily at the subject for a period of more than twenty years, I thought it unlikely that I had overlooked any very obvious structural change in the blood-vessels. What, then, could be the nature of that "hyalin-fibroid" appearance external to the muscular coat of the artery, which is so strikingly displayed in the authors' drawings? I ventured, with some hesitation, to suggest the possibility that the authors had mistaken the appearance of the normal tunica adventitia, when im. mersed in glycerine or dilute acetic acid, for a pathological change; it having been stated in the paper that the specimens were preserved in glycerine and camphor-water. My suggestion naturally enough elicited from the Society some manifestation of surprise. It seemed unlikely that any one would undertake a laborious series of investigations, extending, as Sir William Gull said, over a period of four years, without taking into account the effect of reagents and preservative fluids upon the tissues, whether healthy or morbid. After the discussion was over, and when the meeting had broken up, the few who remained behind had the opportunity of examining some of the specimens which $\mathrm{Dr}$. Sutton had brought with him. A sight of the preparations convinced me at once that my surmise had been correct, and that the so-called "hyalin-fibroid" is neither more nor less than the normal tunica adventitia, probably hypertrophied together with the muscular coat, and certainly distended and rendered transparent by the endosmosis of the highly refracting glycerine fluid. The regular arrangement of the elongated nuclei, with their long diameter in the direction of the arterial canal, and the entire absence of any abnormal appearance, afford conclusive evidence that the tunica adventitia has undergone no pathological change. It is well known that this tunica adventitia is a frequent seat of cell-proliferation in cases of acute tubercular and inflammatory disease ; but no such changes are here in question.

In order still further to test the accuracy of my conclusion, the attention of Sir William Gull and Dr. Sutton was directed to one of my own specimens. Each of them separately and independently expressed his opinion that the arteries in that specimen presented the hyaline appearance which they had described and figured. I then assured them of the fact that the specimen in question was from the pia mater of a sheep believed to have been healthy until killed by the knife of the butcher (see Fig. I). I have similar specimens from the pia mater of

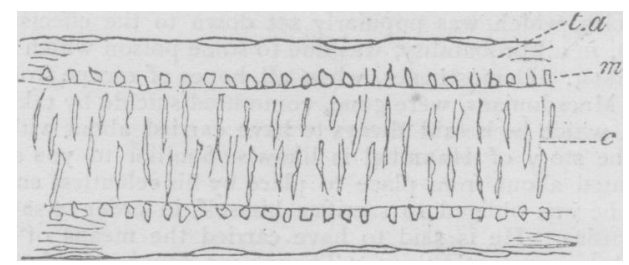

Fig. x.-Artery from the pia mater of a healthy sheep : the tunica adventitia expanded and rendered hyaline by glycerine. $t, a$. Tunica adventitia. $m$. Muscular coat. c. Canal of artery.

a healthy cat ; and I undertake to make similar preparations from the vessels of any healthy animal.

$I$ am indebted to the zeal and energy of my able friend and colleague, Dr. Kelly, for a large number of specimens of arteries, normal and pathological. As a rule, in the specimens which are preserved in gly. cerine or in dilute acetic acid, the arteries have had their tunica adven. 
titia more or less expanded and rendered translucent by the imbibed fluid; and although, without doubt, when the arterial walls undergo true hypertrophy, there is a proportionate increase in the thickness of both the muscular layer and the tunica adventitia, the width and the hyaline appearance of this tunic depend, not upon the degree of its hypertrophy, but upon the extent to which it has become distended by imbibed fluid. This imbibition of fluid may occur as a result of maceration of the vessels in the subarachnoid fluid after death, but it is especially favoured by immersion in glycerine or in dilute acetic acid. In some specimens, so great is this post mortem imbibition of fluid, that the muscular coat may be seen to be irregularly pushed inwards towards the canal of the artery, which consequently appears to be much narrowed. Acetic acid produces the hyaline appearance in the tunica adventitia with extreme rapidity, as may be proved by the following experiment. Place a portion of pia mater on a glass-slide in water or glycerine, and cover it with thin glass in the usual way. The tunica adventitia of the arteries will be seen in close contact with the muscular layer, and having a fibrous appearance, with a wavy outline. Now put a drop of acetic acid at the edge of the covering glass, and allow it to run in, watching the effect beneath the microscope. As the diluted acid comes into contact with the arteries, their external tunic will be seen to swell up and become transparent, while the muscular coat is often irregularly pushed inwards. The vessels thus rapidly assume the appearance which is more slowly produced by glycerine, In this way, a hyaline condition of the tunica adventitia, not to be distinguished from the appearances described and depicted by Sir William Gull and Dr. Sutton, may with ease, and certainty be generated in the course of a few seconds. The appearance of the tunica adventitia, thus acted on by acetic acid, is well represented in a woodcut at p. 486 of Kölliker's well known Manual of Human Microscopic Anatomy, 1860.

While the authors of the paper have based their new theory and nomenclature of Bright's disease upon an erroneous interpretation of the appearances to which $I$ have referred, they entirely ignore the existence of hypertrophy of the muscular coat of the artery, which yet is conspicuously present in many of their own specimens. I showed Sir Wm. Gull a specimen of my own, in which the muscular coat of the artery was about three times the normal thickness for a vessel of that size. He maintained that he saw only a healthy artery. In one sense this was true, since the walls of the artery were composed of only normal tissue, which was, however, excessive in quantity, as Sir Wm. Gull would have seen and admitted if he had accustomed himself, as I have done, to compare the relative thickness of the muscular coat in strictly normal and in hypertrophied arteries (compare Figs. 2 and 3).

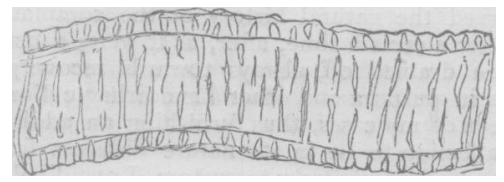

Fig. 2.-Normal artery from the skin.

The authors of the paper maintain that, from some not yet ascertained cause, an arterio-capillary fibrosis in the various tissues throughout the body is the primary condition of Bright's disease. I, on the contrary, agreeing with all modern pathologists that the disease

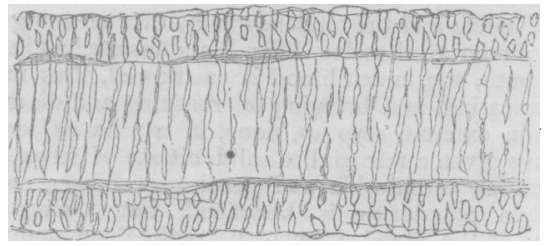

Fig. 3.-Artery from the skin in a case of chronic Bright's disease. The muscular coat hypertrophied.

is always of constitutional origin, believe that the proximate cause of the various forms of Bright's disease is some morbid condition of blood. In the case of the small red kidney, this morbid condition is closely allied to the gouty cachexia, and is often associated with chronic alcoholism. The glandular tissues of the kidney suffer in the discharge of their excretory functions, and the organ wastes and contracts. The blood is then rendered secondarily impure by retained urinary excreta, and it is otherwise altered in its composition. This morbidly changed blood excites unusual contraction of the minute arteries, by which the circulation is impeded; the left ventricle, on the other hand, beats with increased force to drive the blood through the resisting arterioles. The result of this antagonism of forces is that the muscular walls of the minute arteries throughout the body, and those of the left ventricle of the heart, become simultaneously and equally hypertrophied.*

In studying the pathology of the minute arteries, care must be taken to distinguish between hypertrophy of the muscular walls-by which is meant an increase of normal muscular fibre-and the waxy form of degeneration which occurs in the advanced stage of that form of Bright's disease which is associated with the large waxy or lardaceous kidney (see Fig. 4). The arteries in this waxy condition have their walls infil-

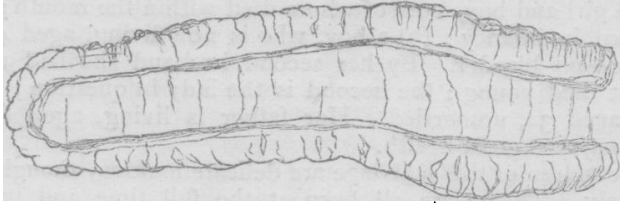

Fig. 4.-Artery from a lardaceous kidney. The walls thickened and waxy.

trated by an albuminous or frbrinous material, which gradually obliterates all trace of normal muscular tissue. Careful observation of this form of arterial degeneration has led me to the conclusion that, as a rule, it supervenes upon the true hypertrophy of the muscular walls; and the gradual transition from one to the other may be readily traced and demonstrated. In like manner, granular and fatty degeneration of the muscular walls of the heart is a common sequence of true hypertrophy.

The specimens to which I have referred in this communication will be exhibited at the conversazione of the Royal Medical and Chirurgical Society on Friday evening, the $7^{\text {th }}$ ipant, when an opportunity will be afforded to compare my specimens with those of Sir Wm. Gull and Dr. Sutton. If the result of that comparison be not deemed conclusive by the majority of the Fellows and their visitors, I would suggest that the President and Council of the Society appoint a Scientific Committee to examine and report upon the specimens. As Sir Wm. Gull remarked after the discussion on the 28th ultimo, our object in this inquiry is not victory in controversy, but the establishment of truth.

\section{O N H EM OPH I L A .}

By J. WEST WALKER, M.B.Lond., Spilsby.

THE following family history is interesting, as furnishing examples of Hæmophilia, a disease which has reoently been brought prominently before the profession in this country Dr. Wickham Legg. I intimately knew the father and mother anterior to their marriage eleven years ago; and both before and since that event have been their constant medical attendant. I fail to trace any facts bearing upon the important question of hereditary character, and am inclined to think the present an instance illustrative of the first start of a pathological condition which, there is every reason to fear, will be trans. mitted to descendants. The ultimate cause of the phenomena is involved in obscurity. As, on the one hand, some, so to speak, unhealthy selection seems to bring about the development of this singular morbid process-so, on the other, a more healthy selection, through more or fewer intermarriages, tends to affect its diminution and ultimate eradication. Hence, notwithstanding the hereditary tendency is a most important and diagnostic feature, we often fail, happily, in our endeavours to trace it in full force through more than a limited number of generations.

The father is a clergyman of the Church of England, and master of a grammar school. He is very tall -6 feet 4 inches-and thin. He is a hard-working and somewhat delicate looking man, and carries with him the appearance of worry and anxiety. He has never since I have known him had any serious illness, but he occasionally suffers from headaches, which find relief in epistaxis. His age at marriage was thirty-nine. He is no relation to his wife, and he never heard of a bleeder in his family. His father, a clergyman, died at the age of sixty-two of heart-disease; his mother at the age of seventy-four of general decay. She was a remarkably healthy active woman up to seventy, when she experienced an attack of hæmorrhage (hæmatemesis?), which left her weakly and with health impaired during the

* For a more detailed explanation of the physiology of the cardio-arterial hypertrophy, reference may be made to my paper in the 5 ist volume of the Medico Chirurgical Transactions; and to a paper in the BRITISH MBDICAL Jourarar. Chirurgical Tras
April $16 \mathrm{th}, 1870$. 